\title{
Normosensorisches Spätschielen: Differenzialdiagnose und Therapie
}

\section{O. Ehrt}

Augenklinik der Ludwig-Maximilians-Universität, München

Beim normosensorischen Spätschielen handelt es sich um eine meist im 3. und 4. Lebensjahr, ohne erkennbare okuläre oder neurologische Ursache akut auftretende, oft initial intermittierend beginnende konkomitante Esotropie. Angaben des Patienten oder die Beobachtung seines Verhaltens zeigen eine wenige Tage dauernde Diplopie, da die bisherige Binokularentwicklung regelrecht war.

Differenzialdiagnostisch muss eine akkommodative Esotropie ( $<4$ dpt Hyperopie, Winkelreduktion cc), ein dekompensierter Mikrostrabismus (Amblyopie, meist keine Diplopie, schon zu Beginn ARC), eine sekundäre Esotropie (organische Visusminderung, RAPD) und eine ZNS-Erkrankung
(Cephalgie, Erbrechen, verhaltensauffällig, systemische Motorik- oder Koordinationsstörung, okuläre Motilität, STP) ausgeschlossen werde. Eine Bildgebung u. a. invasive Diagnostik sollte nur durchgeführt werden, wenn entsprechende klinisch neurologische und okuläre Auffälligkeiten gefunden werden.

Die Therapie des normosensorischen Spätschielens besteht in einem sofortigen Refraktionsausgleich, der Amblyopietherapie und einem alternierenden Prismenfolienausgleich. Eine Operation sollte dann innerhalb von 6 Monaten erfolgen, da ein längeres Zuwarten, die Prognose für das räumliche Sehen verschlechtert. Postoperativ sollte jeder Restwinkel mit Prismen bzw. Re-Op korrigiert werden.

\section{Strabismus divergens - einmal anders Augenstellung, Augendarstellung und Blickrichtigung in der Bildenden Kunst E. Stangler-Zuschrott \\ Wien \\ Technische Bearbeitung: Reinhard Müller, Wien}

Bei Besichtigung religiöser Kulturstätten fällt häufig Divergenzschielen der plastischen Figuren auf, besonders im späten Mittelalter und im Barock. Die dargestellten Figuren schielen vorwiegend nach außen und unten. Als Erklärung von Kunsthistorikern war bisher nur religiöse Ekstase oder der ,vergeistigte Blick“ bekannt, dies vorwiegend beim Blick nach oben. Auf der Suche nach weiterem Verständnis und Besichtigung von vielen Museen und Kirchen, findet man von der Antike bis zum Ende der Romantik Divergenzschielen nur im Zusammenhang mit Tod und Grabbeigaben.

Entsprechend dem Zeitgeist der Mystik und mangelnder anatomischer Kenntnisse werden im Mittelalter und Barock

\section{Indikationen für Ein-Muskel-Operationen}

\section{K.-P. Boergen}

Augenklinik des Klinikums der Universität München, München

Zusammenfassung. Kombinierte Augenmuskeloperationen stellen die häufigsten strabologischen Eingriffe dar. Hierdurch wird die Muskelspannung nicht wesentlich verändert. Gelegentlich gibt es aber auch Indikationen, bei denen eine Spannungsveränderung durch Operation an nur einem Augenmuskel günstig und daher sinnvoll sein kann. Dies ist z. B. bei primären Überfunktionen wie beim Strabismus sursoadductorius oder deorsoadductorius der Fall, bei denen Rücklagerungen des überfunktiven Muskels gute funktionelle Ergebnisse aufweisen. Auch bei mechanischen Bewe- dem Blick der Figuren Emotionen aller Arten zugeordnet, sowohl im positiven als auch im negativen Sinn. Daher müssen solche Plastiken in ihrer Gesamtheit und Umgebung betrachtet werden. Bei Aufhebung des Binokularsehens kann eine Statue zwei verschiedene Objekte ansehen. Weiters sollte die vom Künstler vorgesehene Stellung der Figur im Raum berücksichtigt werden, Schielen fällt dann oft nicht auf. Je nach Künstlerpersönlichkeit und Werkstätte wird Strabismus bevorzugt dargestellt oder eher selten. Konvergent schielende Heiligenstatuen sind extrem selten (Heilige Ottilie). gungseinschränkungen wie der endokrinen Orbitopathie sind schwächende Eingriffe an nur einem Muskel vorzuziehen. Schwächende oder stärkende Operationen an einem Augenmuskel sind auch dann indiziert, wenn der Schielwinkel für ein kombiniertes Vorgehen zu klein ist. Ferner soll auf die Stärkung von Teilfunktionen einzelner Muskel hingewiesen werden, wie die Vorderrandverlagerung der Obliquussuperior-Sehne im Rahmen der operativen Therapie der Trochlearisparese. 